\title{
Design of a Fully Differential Power Output Stage for a Class D Audio Amplifier Using a Single-Ended Power Supply
}

\author{
Pedro V. Leitão, João L.A. de Melo, and Nuno Paulino \\ Departamento de Engenharia Electrotécnica (DEE), UNINOVA/CTS \\ Faculdade de Ciências e Tecnologia (FCT)-Universidade Nova de Lisboa (UNL) \\ 2829-516, Caparica, Portugal \\ pvicenteleitao@gmail.com, research@joaodemelo.com, \\ nunop@uninova.pt
}

\begin{abstract}
This paper presents a full-bridge discrete power output stage for a Class D audio amplifier using a single-ended power supply. The circuit receives a digital control signal with $5 \mathrm{~V}$ of amplitude and it generates a floating differential output voltage up to $20 \mathrm{~V}$ of amplitude from a single $20 \mathrm{~V}$ power supply voltage. Using as control signal a pulse density modulation (PDM) wave generated by an optimized $3^{\text {rd }}$ order continuous-time (CT) sigma delta modulator $(\Sigma \Delta \mathrm{M})$, the system achieves a signal-to-noise-plus-distortion ratio (SNDR) of $83.1 \mathrm{~dB}$, total harmonic distortion (THD) of $-89 \mathrm{~dB}$ and a power efficiency of $92 \%$, while delivering $11 \mathrm{~W}$ over an $8-\Omega$ load with a signal bandwidth of $20 \mathrm{kHz}$ and a sampling frequency of $1.28 \mathrm{MHz}$.
\end{abstract}

Keywords: Class D Audio Amplifier, Output Stage, Gate Driver.

\section{Introduction}

There has been an ongoing tendency to increase the energy efficiency of every electronic device as global sustainability starts to be a major concern. Audio amplifiers are not different as they play a big role regarding power consuming in electronic systems. In Class D amplifiers the output stage devices operate as switches, resulting that when the CMOS output transistors are conducting there is a small drainto-source voltage drop causing the dissipated power by them to be close to zero. Comparing with the typical Class $\mathrm{AB}$ amplifier, which has a maximum theoretical efficiency of $78.5 \%$, the Class D amplifier can theoretically achieve a power efficiency of $100 \%$ [1].

The performance of a Class D audio amplifier output stage is mainly determined by the output stage configuration and the quality of the output switching [2]. The fullbridge configuration versus the half-bridge configuration can cancel out even order harmonic distortion, DC offset, allows the use of a single power supply (which doubles the available power supply voltage) and the use of a 1.5-bit quantization scheme. This quantization scheme will provide current to the load only when needed, decreasing the switching activity thus causing the power efficiency to increase while 
decreasing the electromagnetic interference (EMI) [3]-[4]. The output power transistors connect the output nodes either to the positive power supply or ground, therefore it is important to guaranty that they do not conduct simultaneously, in order to prevent large current spikes from the positive power supply to ground from occurring. This results in a need to introduce non-overlapping time which is called dead time in the control signals and is one of the most dominant sources of distortion in Class D audio amplifiers [6]. In general, in a Class D output stage, the power output transistors are implemented using two identical NMOS transistors in a totempole configuration. These are preferred due to their small area and capacitance. Nonetheless, in order to drive the high side NMOS power transistor it is necessary additional circuitry using more complicated techniques (e.g. bootstrap technique) and timed circuitries in order to correctly activate the necessary transistors [7]-[9].

The proposed output stage presented in this paper, depicted in Fig. 1, relies on a full-bridge configuration and in a PMOS-NMOS output totem-pole arrangement which enables the high side gate driver to have a simpler circuitry. The goal is to achieve a THD close to $-90 \mathrm{~dB}(0.0032 \%)$ and power efficiency higher than $90 \%$ while using a single $20 \mathrm{~V}$ power supply over an $8-\Omega$ load.

The system behavior is as follows. The analog input signal is modulated into a digital signal through pulse-width modulation (PWM) or pulse-density modulation (PDM), then this signal is level-shifted in order to drive the output CMOS power transistors and finally the differential output voltage is filtered by a low-pass filter, which reconstructs the signal before the loudspeaker in order to eliminate the high frequency content. The non-overlapping time module provides the necessary time delay in order to prevent simultaneous conduction between the output transistors. The modulation part of the system has a feedback from the output stage which enables the system to correct the output stage errors and to increase the power supply rejection ratio.
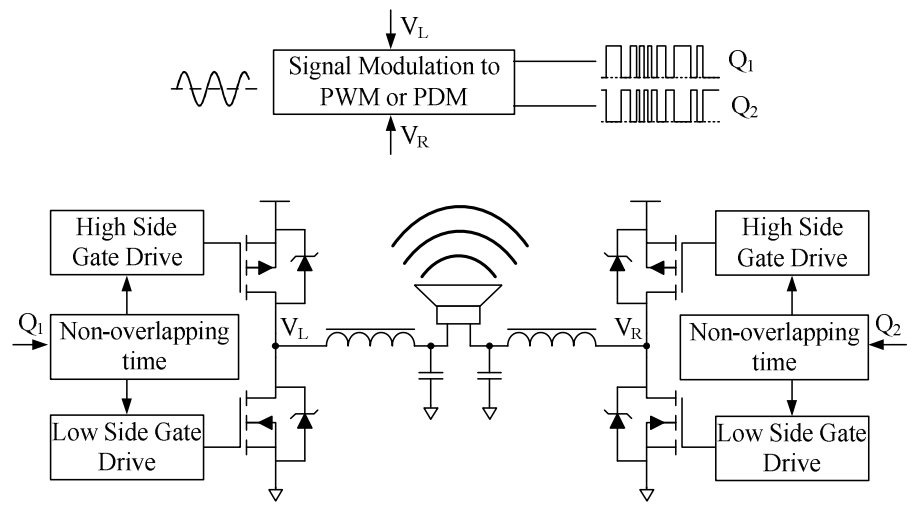

Fig. 1. Class D audio amplifier with full-bridge configuration

\section{Relationship to Internet of Things}

The Internet of Things refers to uniquely identify objects and their virtual representations in an Internet-like structure. If all objects and people in daily life were 
equipped with radio tags, they could be easily identified and inventoried by computers. In a social context, audio amplifiers, especially Class D amplifiers, being high efficiency are able to aid visual conditioned people in their day-to-day living by identifying the object/person as they come by with an extended life battery.

\section{Class D Audio Amplifiers Output Stage}

\subsection{Low Side Gate Driver}

The power output NMOS transistor gate can be simply driven by a CMOS inverter. Controlling the inverter switching with timed pulses can significantly decrease the power consumption. Fig. 2(a) shows the proposed low side driver and Fig. 2(b) shows the simulated voltages and currents in the circuit.

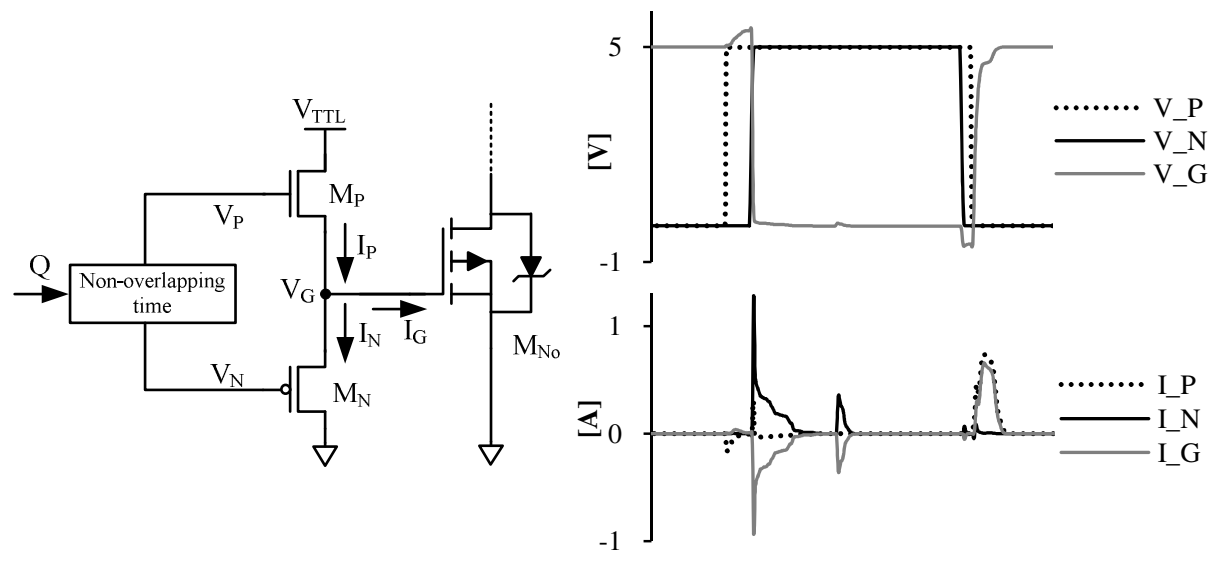

(a)

(b)

Fig. 2. (a) Low side gate driver and power NMOS transistor (b) Simulated voltage levels and current flow in the low side gate driver during the $\mathrm{M}_{\mathrm{N} 0}$ switching

In order to achieve high efficiency the drain current of $\mathrm{M}_{\mathrm{P}}\left(I_{P}\right)$ and $\mathrm{M}_{\mathrm{N}}\left(I_{N}\right)$ should only flow to the gate of $\mathrm{M}_{\mathrm{N} 0}\left(I_{G}\right)$. This can be achieved by using the correct nonoverlapping value, as represented in Fig. 2(b). Otherwise, a large current can flow from the power supply through the inverter $\left(\mathrm{M}_{\mathrm{P}}\right.$ and $\left.\mathrm{M}_{\mathrm{N}}\right)$.

\subsection{High Side Gate Driver}

The starting point for this circuit was the PMOS gate driver described in [10] and depicted in the box of Fig. 3(a). The circuit operation is as follows: when $\mathrm{P}_{1}$ is high the transistors $\mathrm{M}_{1}$ and $\mathrm{M}_{2}$ are on, forcing the base-to-emitter voltage drop of transistor $\mathrm{Q}_{1}$ to be zero, turning it off. $\mathrm{M}_{2}$ will also push-down the gate voltage of transistor $\mathrm{M}_{\mathrm{PLeft}}$ to zero, turning it on. When $\mathrm{P}_{1}$ is low, the transistor $\mathrm{Q}_{1}$ will turn on due to the resistor $R_{1}$ which will pull-up the gate voltage of $M_{\text {PLeft }}$. 
This means that the $\mathrm{M}_{\text {PLeft }}$ turn-off speed is dependent on $\mathrm{R}_{1}$ which imposes a severe trade-off: when $\mathrm{M}_{\text {PLeft }}$ is on, a DC current $V_{C C} /\left(R_{l}+R_{D S(M I)}\right)$ will flow through $\mathrm{R}_{1}$ and $\mathrm{M}_{1}$, creating an undesired power dissipation. Increasing $\mathrm{R}_{1}$ in order to reduce this current will slow down the $\mathrm{M}_{\mathrm{PLeft}}$ turn-off speed as this resistor bias $\mathrm{Q}_{1}$. This classifies the gate drive as Class A when the $\mathrm{M}_{\mathrm{PLeft}}$ is off, as it is necessary to have a constant DC current.

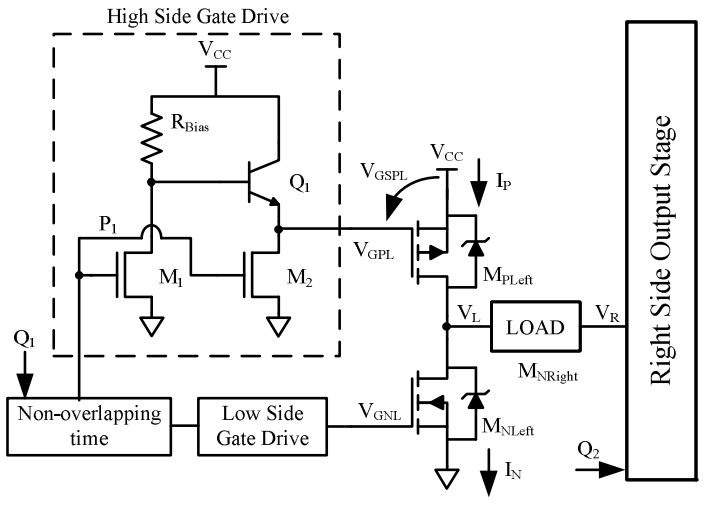

(a)

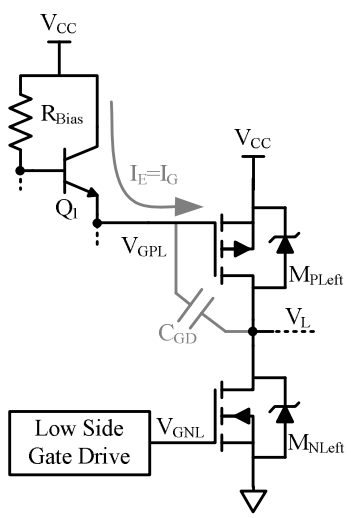

(b)

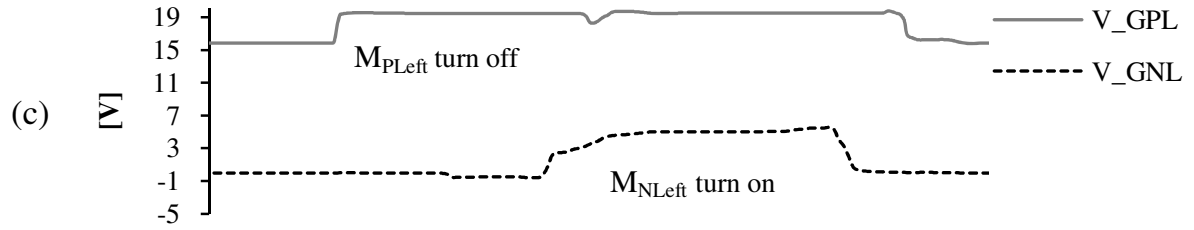

(d)

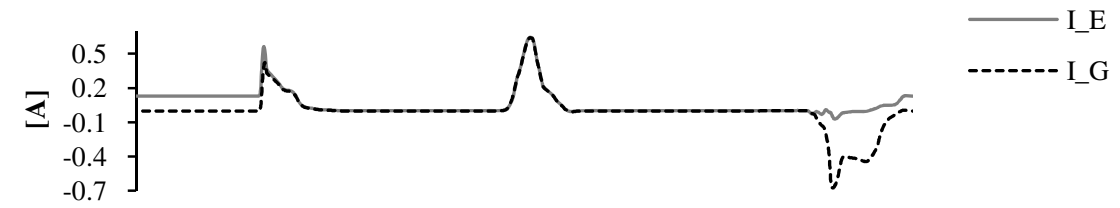

(f) $\begin{array}{r}3 \\ 2 \\ 1 \\ 0 \\ -1\end{array}$
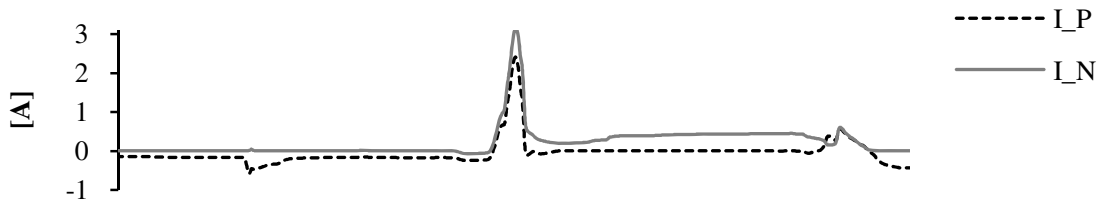

Fig. 3. (a) PMOS gate driver [10] (in box) in full-bridge configuration (b) PMOS gate driver [10] representation with parasitic capacitance while $\mathrm{M}_{\mathrm{NLeft}}$ turn on and an example showing the simulated (c) $V_{G P L}$ and $V_{G N L}$ voltages (d) $I_{E}$ and $I_{G}$ currents (f) $I_{P}$ and $I_{N}$ currents

By inspecting the circuit it is possible to see that the $\mathrm{M}_{\mathrm{PLeft}}$ gate-to-source voltage, $V_{G S P L}$, during $\mathrm{M}_{\mathrm{PLeft}}$ turn-off, is equal to $V_{R \text { Bias }}+V_{B E(Q 1)}$. This means that $\mathrm{M}_{\mathrm{PLeft}}$ is not fully turned off as $V_{G S P L}$ is not exactly zero. Fig. 3(b) shows the high side gate driver 
with parasitic capacitances during the $\mathrm{M}_{\mathrm{PLeft}}$ turn off and $\mathrm{M}_{\mathrm{NLeft}}$ turn on, and on Fig. 3(c), (d) and (f) an example with the simulated transitory waves is also shown. As $\mathrm{M}_{\mathrm{PLeft}}$ is turning off, $V_{G P L}$ value increases to $V_{C C}-V_{R I}+V_{B E}$ which in the shown example is $19.5 \mathrm{~V}$, with a $20 \mathrm{~V}$ power supply. After the non-overlapping time the $V_{G N L}$ voltage increases to $5 \mathrm{~V}$ turning $\mathrm{M}_{\mathrm{NLeft}}$ on. This will pull down the node $V_{L}$, forcing the $V_{G P L}$ node also to be pulled down, due to the parasitic capacitance $\mathrm{C}_{\mathrm{GD}}$ of $\mathrm{M}_{\mathrm{PLeft}}$ being large (as represented in Fig. 3(b)). This will cause the $V_{G S P L}$ voltage to increase, falsely turning $\mathrm{M}_{\mathrm{PLeft}}$ on and creating large current spikes between $V_{C C}$ and ground through transistors $\mathrm{M}_{\mathrm{PLeft}}$ and $\mathrm{M}_{\mathrm{NLeft}}$ because they are simultaneously conducting. This effect is called shoot-through current [5] and will decrease the efficiency significantly, introduce harmonic distortion and even destroy the output transistors if $\mathrm{Q}_{1}$ takes too long to respond to this variation.

This problem is bypassed by the proposed high side gate driver presented in this paper.

\subsection{Proposed High Side Gate Driver}

The new full-bridge output stage proposed in this paper is presented in Fig. 4. Two CMOS inverters were placed at the gate of the output PMOS. In order to achieve lower power dissipation, especially in $\mathrm{R}_{\mathrm{Bias}}$, one zener diode was inserted in each branch of the circuit. The zener voltage of these diodes should be $V_{C C}-5 \mathrm{~V}$ so that the $V_{G S}$ voltage drop of the output PMOS transistor, the $\mathrm{R}_{\text {Bias }}$ voltage drop and the CMOS inverters only varies from $5 \mathrm{~V}$ to $V_{C C}$. This will cause the $V_{G S}$ voltage drop of the PMOS to be constant thus improving the performance of the circuit while simplifying the voltage supply scaling, as only the zener diode needs to be scaled.

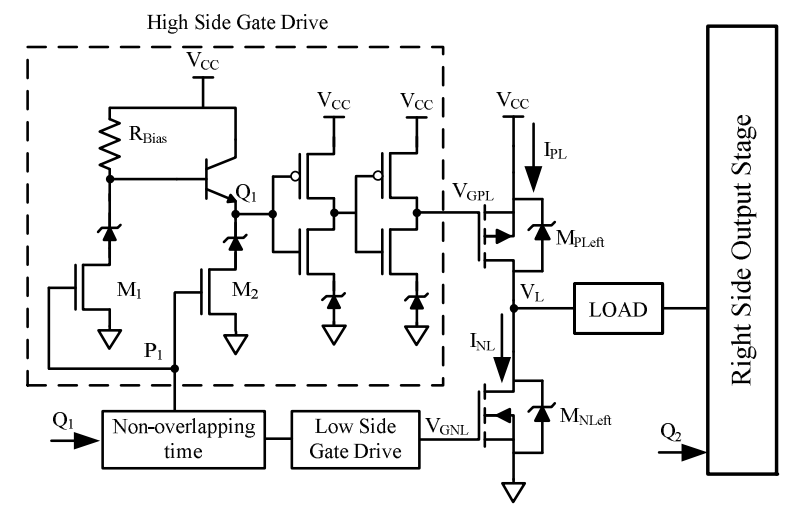

Fig. 4. Proposed PMOS gate driver (in box) in full-bridge configuration

The inverters placed at the gate of the output PMOS will correctly pull-up $V_{G P L}$ to $V_{C C}$ so that it will be correctly turned off. The correctly timed delay will prevent shoot-through current. 
An example for the switching waves of the output stage is shown on Fig. 5. The circle in Fig. 5(b) shows a commonly found effect in Class D output stages. As $V_{L}$ voltage rises to $V_{C C}$, a parasitic current goes from $V_{C C}$ through the parasitic capacitance $\mathrm{C}_{\mathrm{GD}}$ of the NMOS power transistor. This effect is rapidly compensated by the low side gate driver.

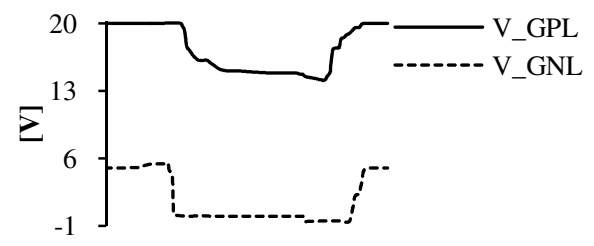

(a)

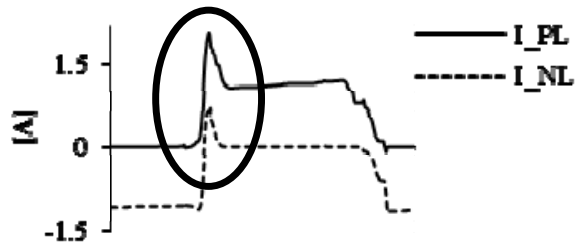

(b)

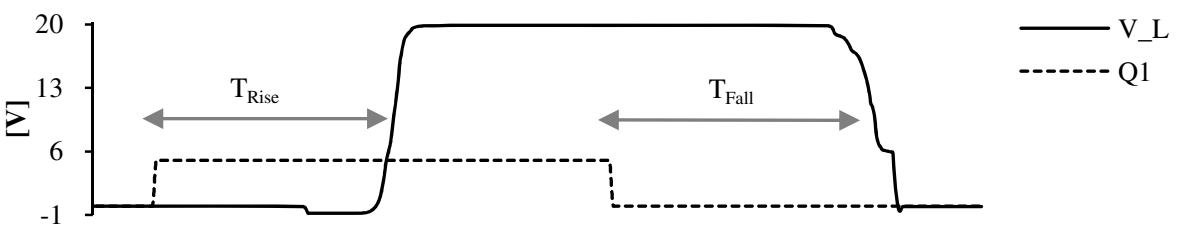

(c)

Fig. 5. Simulated example for (a) switching voltage levels at the gate of the output power transistors (b) current in the output power transistors (c) input voltage $\left(Q_{l}\right)$ and output voltage $\left(V_{L}\right)$ with the $\mathrm{T}_{\text {Rise }}$ and $\mathrm{T}_{\text {Fall }}$ delay represented

\section{Class D Audio Amplifier with a $3^{\text {rd }}$ Order 1.5-Bit Continuous-Time (CT) Sigma Delta $(\Sigma \Delta)$ Modulator}

The presented output stage was simulated with a $3^{\text {rd }}$ order 1.5 bit $\Sigma \Delta \mathrm{M}$ optimized for Class D audio amplifiers with distributed feedback and local resonator feedback, described in [11]. The output stage was design to handle $20 \mathrm{~V}$ power supply, $20 \mathrm{kHz}$ low-pass filter, a sampling frequency of $1.28 \mathrm{MHz}$ and to drive an $8-\Omega$ load.

Fig. 6(a) shows the $P_{\text {RBias }}$ dissipation plot during continuous conduction and the Fig. 6(b) shows the $\mathrm{T}_{\text {Rise }}$ and $\mathrm{T}_{\text {Fall }}$ delay of the output stage with the $\mathrm{R}_{\text {Bias }}$ increase. From these plots it is possible to conclude that the output stage power efficiency will increase as $\mathrm{R}_{\mathrm{Bias}}$ increases, but the $\Sigma \Delta \mathrm{M}$ will have to handle the output stage increased delay. The used $3^{\text {rd }}$ order $\Sigma \Delta \mathrm{M}$ was optimized using $100 \mathrm{~ns}$ and $150 \mathrm{~ns}$ delay while using the same goal function. This allowed the use of $R_{\text {Bias }}$ values between 60 to 100 $\Omega$ and 160 to $200 \Omega$, respectively. Table 1 shows the system performance summary for these optimizations and Fig. 7 shows the fast Fourier transform (FFT) of the output sinusoidal wave while applying a $0 \mathrm{dBV}$ and $2 \mathrm{kHz}$ input signal. 
(a)

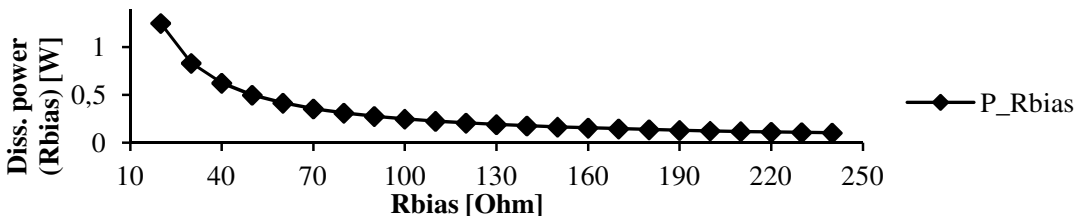

(b)
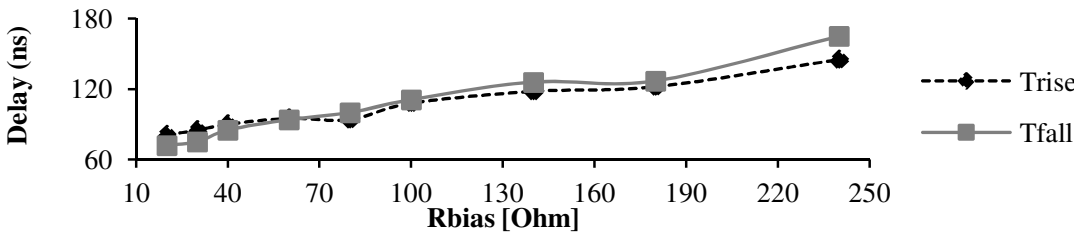

Fig. 6. (a) $P_{\text {Rbias }}$ plot $\left(P_{\text {Rbias }}=5^{2} / R_{\text {Bias }}\right)$ (b) $\mathrm{T}_{\text {Rise }}$ and $\mathrm{T}_{\text {Fall }}$ delay

Table 1. System performance summary with $100 \mathrm{~ns}$ output stage delay optimization (\#1) and 150 ns delay optimization (\#2)

\begin{tabular}{cccccccccc}
\hline \multirow{2}{*}{ Optm. $\mathrm{R}_{\text {Bias }}(\Omega)$} & SNDR $(\mathrm{dB})$ & $\mathrm{THD}(\mathrm{dB})$ & $\mathrm{HD} 2(\mathrm{~dB})$ & $\mathrm{HD} 3(\mathrm{~dB})$ & $\eta(\%)$ & $\mathrm{P}_{\text {Load }}(\mathrm{W})$ & \multicolumn{2}{c}{ Delay $(\mathrm{ns})$} \\
\hline \multirow{3}{*}{$\# 1$} & 60 & 82.21 & -84.96 & -90.3 & -89.3 & 87.90 & 13.36 & 100 & 95 \\
& 80 & 81.92 & -84.76 & -90.8 & -88.8 & 89.21 & 13.36 & 105 & 100 \\
& 100 & 83.09 & -86.38 & -93.1 & -89.2 & 89.76 & 13.36 & 113 & 110 \\
\hline \multirow{3}{*}{$\# 2$} & 160 & 83.62 & -89.70 & -98.4 & -101.5 & 91.73 & 11.29 & 125 & 135 \\
& 180 & 83.41 & -87.08 & -97.1 & -100.4 & 91.69 & 11.30 & 135 & 140 \\
& 200 & 83.14 & -88.94 & -99.9 & -106.3 & 92.20 & 11.30 & 140 & 150 \\
\hline
\end{tabular}

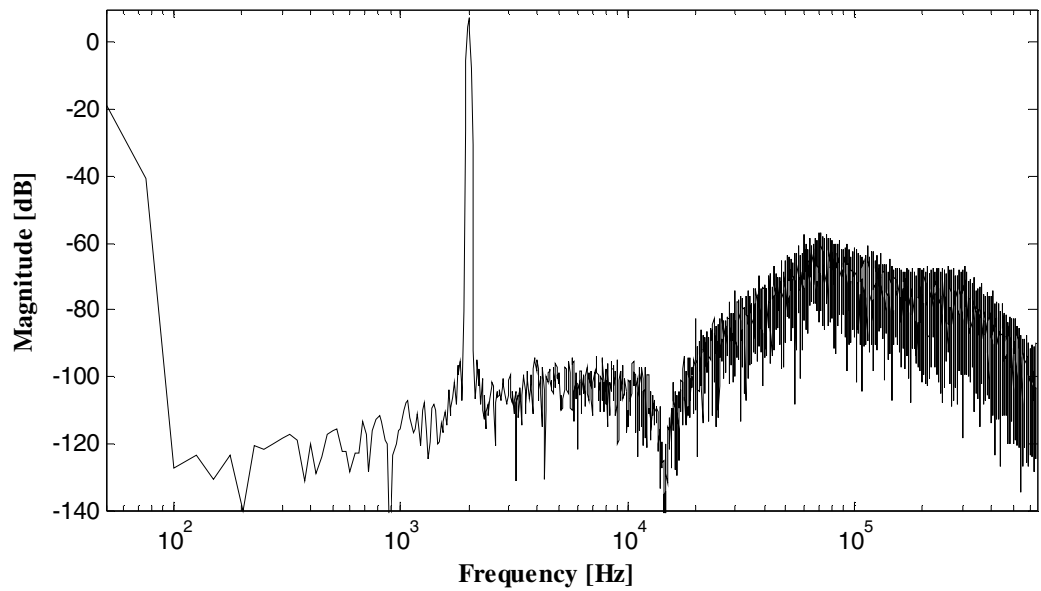

Fig. 7. Spectrum of the output signal (using a Blackman-Harris window and $2^{16}$ points) with $\mathrm{R}_{\text {Bias }}$ equal to $200 \Omega$ and SNDR of $83 \mathrm{~dB}$ 
These results show that using a higher $\mathrm{R}_{\mathrm{Bias}}$ value the system will operate with higher power efficiency, higher SNDR and lower harmonic distortion, but will deliver less energy to the output load. This is due to the increased $\mathrm{T}_{\text {Rise }}$ and $\mathrm{T}_{\text {Fall }}$ delay which will result in a smaller active time in the output PDM wave, thus reducing the maximum output power. The best compromise between SNDR, harmonic distortion and power efficiency has been found to be with $\mathrm{R}_{\mathrm{Bias}}$ equal to $200 \Omega$ where the system achieves a SNDR of $83 \mathrm{~dB}$, THD of $-89 \mathrm{~dB}$ and power efficiency $\left(\mathrm{P}_{\text {Load }} / \mathrm{P}_{\text {delivered }}\right)$ of $92 \%$.

\section{Conclusions}

A full-bridge discrete power output stage for a Class D audio amplifier using a single $20 \mathrm{~V}$ power supply voltage was presented. The circuit is controlled by a digital $5 \mathrm{~V}$ of amplitude PDM signal, produced by an optimized $3^{\text {rd }}$ order $\sum \Delta$ modulator with the output stage inside the closed loop. This system is capable of driving an $8-\Omega$ load while achieving a SNDR of $83 \mathrm{~dB}$ and a THD of $-89 \mathrm{~dB}(0.0035 \%)$. The use of a PMOS-NMOS power output inverter simplified the design while still achieving power efficiency of $92 \%$ for an $11.3 \mathrm{~W}$ power delivered to the load. The use of 1.5-bit quantization scheme and the lossless LC low-pass filter allowed to reduce the EMI distortion (max. of $-60 \mathrm{~dB}$ at $80 \mathrm{kHz}$ ).

\section{References}

1. Duncan, B.: High Performance Audio Power Amplifiers, Newnes, Great Britain (1996)

2. Berkout, M.: A Class D Output Stage with Zero Dead Time. In: Proc. IEEE Solid-State Circuits Conference (ISSCC), pp. 134-135 (2003)

3. Pan, J., Yao, L.B., Lian, Y.: A Sigma-Delta Class D Audio Power Amplifier in 0.35um CMOS Technology. In: Int. SoC Design Conference, pp. 24-25 (2008)

4. de Melo, J., Paulino, N.: Design of a 3rd order 1.5-bit continuous-time (CT) Sigma-Delta (SD) modulator optimized for Class D audio power amplifier. J. Microelectronics and Computer Science 1(2) (2011)

5. Gaalaas, E.: Class-D audio amplifiers: what, why, and how. Analog Dialogue 40 (2003); Analog Devices

6. Mosely, I., Mellor, P., Bingham, M.: Effect of Dead Time on Harmonic Distortion in Class D Audio Power Amplifiers. Electronics Letters 35(12), 950-952 (1999)

7. Morrow, P., et al.: A 20-W Stereo Class D Audio Output Stage in $0.6 \mathrm{~mm}$ BCDMOS Technology. IEEE J. Solid-State Circuits 39(11), 1948-1958 (2004)

8. Berkhout, M.: An Integrated 200-W Class-D Audio Amplifier. IEEE J. Solid-State Circuits 38(7), 1198-1206 (2003)

9. Berkhout, M., Dooper, L.: A 3.4W Digital-In Class D Audio Amplifier. In: Proc. ESSCIRC (ESSCIRC), pp. 87-90 (2011)

10. Yi, H., Hong, S.: Design of L-band high speed pulsed power amplifier using ldmos fet. In: Progress In Electromagnetics Research M, vol. 2, pp. 153-165 (2008)

11. de Melo, J.L.A., Nowacki, B., Paulino, N., Goes, J.: Design methodology for sigma-delta modulators based on a genetic algorithm using hybrid cost functions. In: Proc. IEEE Int. Symp. Circuits Systems (ISCAS), pp. 301-304 (May 2012) 